\title{
A comparison of fabrication techniques for hollow retroreflectors
}

\author{
Alix Preston, Stephen Merkowitz ${ }^{\mathrm{a}}$ \\ ${ }^{a}$ NASA, Goddard Space Flight Center, 8800 Greenbelt Rd, Greenbelt, MD, USA, 20771
}

\begin{abstract}
Despite the wide usage of hollow retroreflectors, there is limited literature involving their fabrication techniques and only two documented construction methods could be found. One consists of an adjustable fixture that allows for the independent alignment of each mirror, while the other consists of a modified solid retroreflector that is used as a mandrel. Although both methods were shown to produce hollow retroreflectors with arcsecond dihedral angle errors, a comparison and analysis of each method could not be found which makes it difficult to ascertain which method would be better suited to use for precision-aligned retroreflectors. Although epoxy bonding is generally the preferred method to adhere the three mirrors, a relatively new method known as hydroxide-catalysis bonding (HCB) presents several potential advantages over epoxy bonding. HCB has been used to bond several optical components for space-based missions, but has never been applied for construction of hollow retroreflectors. In this paper we examine the benefits and limitations of each bonding fixture as well as present results and analysis of hollow retroreflectors made using both epoxy and HCB techniques.
\end{abstract}

Keywords: optics, hollow retroreflectors, fabrication techniques, hydroxide-catalysis bonding, epoxy.

Address all correspondence to: Stephen Merkowitz, NASA, Goddard Space Flight Center, 8800 Greenbelt Rd., Greenbelt, MD, USA; Tel: +1 301-286-9412; E-mail: stephen.m.merkowitz@nasa.gov

\section{Introduction}

A retroreflector is an optical component that uses three mutually perpendicular surfaces to reflect light back to its source such that the incoming beam is parallel to the reflected beam. For the typical retroreflector with mutually perpendicular surfaces, light is reflected back to the source in a parallel manner regardless of the orientation of the retroreflector. This property makes retroreflectors extremely useful for alignment, metrology, laser ranging, and other systems in which the light source or other optics are in motion ${ }^{1}$.

Retroreflectors are often referred to as "corner cubes" and come in the solid and hollow variety. In the case of the solid corner cube, light passes through a piece of glass and is reflected at the back surfaces by either total internal reflection or through reflective coatings. For hollow retroreflectors the light simply reflects off the cube surfaces and does not typically pass through 
any solid material. This configuration presents many advantages over its solid counterpart including eliminating chromatic aberration and material absorption, the potential for a lighter weight optic, and the ability to be constructed out of a variety of materials.

Although hollow retroreflectors are used in a variety of applications, the literature containing their construction methods is minimal and only two methods could be found ${ }^{1-4}$. Although Ref. 4 is the most complete reference that could be found pertaining to both the optical fabrication and mirror mounting techniques, it lacked the specific details needed to fabricate a hollow retroreflector (such as what epoxy to use, details about the bonding fixture, the hydroxide bonding technique, and the construction method to name a few) that are presented here. One method uses a fixture that allows for the independent adjustment of each mirror, while the other method relies upon using a solid corner cube as a mandrel that the mirrors are placed on top of and then bonded into place. Each method has its advantages and disadvantages although a comparison of the two methods could not be found making it difficult to know which method would be potentially better to use for precision-aligned corner cubes. Here we bridge this knowledge gap by comparing corner cubes made using both types of bonding fixture.

While epoxy is generally the preferred method to adhere the mirrors, a relatively new bonding technique known as hydroxide-catalysis bonding (HCB) has potential advantages over epoxy bonding. HCB was originally developed for the Gravity Probe B mission to bond the star tracking telescope but has since found use in several other materials and applications ${ }^{5-8}$. Its high strength, thin bond, and low coefficient of thermal expansion (CTE) make it ideal for a wide range of precision-bonded applications, especially for space based missions and optical components that will operate at low temperatures ${ }^{9-11}$. For this reason, both epoxy and HCB 
methods were used to construct hollow corner cubes and an analysis of both techniques is presented in this paper.

\section{Bonding Fixtures}

Two types of bonding fixtures were investigated and built for constructing hollow retroreflectors: an adjustable fixture and a solid corner cube used as a mandrel. In this section we provide an overview of these two approaches.

\subsection{Adjustable Fixture}

The adjustable fixture consists of three panels that allow one axis of each panel to be adjusted through the use of a threaded screw or micrometer (see Fig. 1a). These panels are held in place using springs and ball bearings that are mounted on a rigid backing structure similar to a common mirror mount. The fixture used in this paper is similar to the fixture used in Ref. 1, but instead of two mirror mounts being used, three mutually perpendicular mirror mounts were attached to a backing/support structure. In this manner the entire fixture could be picked up and moved while still being able to independently adjust each of the fixture panels.

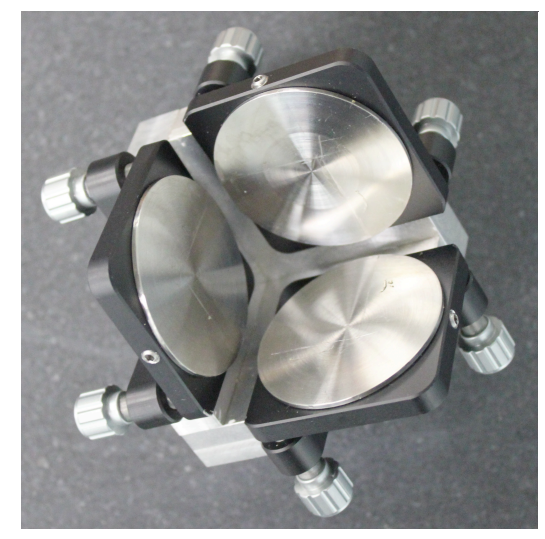

(a)

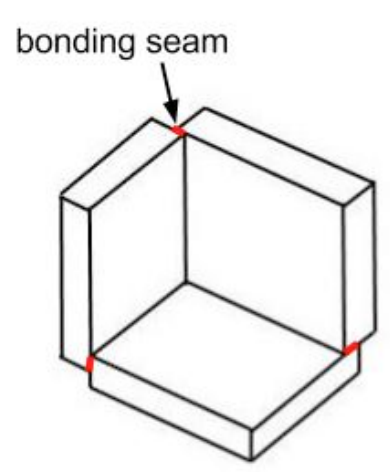

(b) 
Fig. 1 (a) Concept of the adjustable fixture, and (b) an illustration of the bonding seam when epoxy was used as a bonding agent.

There is a gap approximately $1.3 \mathrm{~mm}$ between adjacent panels that allow the independent movement of each panel while still allowing shims to be placed between panels if necessary. The shims were rectangular aluminum bars that had the square dimension equal to the thickness of the mirror minus the thickness of the bonding seam (12.7 mm minus $1.27 \mathrm{~mm}$ with machining tolerances of $0.127 \mathrm{~mm}$ ), and had a length of approximately $76.2 \mathrm{~mm}$. These shims were used to control the overlap of the bonding seam. Glass spacers can also be epoxied to the panels if the full thickness of the mirror needed to be used. Our initial fixture was made out of aluminum and stainless steel, but other materials such as ULE or Zerodur were considered to provide added dimensional stability to the fixture.

To bond the mirrors together, the fixture, shims, and the back of the mirrors were cleaned with acetone. The shims were then put in the corners of the fixture and the mirrors placed on top. The mirrors were orientated such that the bonding face of one mirror sat on the mirrored surface of the next mirror (see Fig. 1b). An initial alignment of the mirrors was done in the fixture and a Verifire XPZ (Zygo Corp.) interferometer was used to measure the dihedral angle errors, wavefront, and maximum beam deviation of the retroreflector ${ }^{12}$. Once the mirrors had been properly aligned under the interferometer, a small amount of epoxy (such as Urelane or Urethane) was applied in small drops along the seams where the mirror and adjustment panel meet. This will hold the mirrors in place while allowing the mirrors to be adjusted as they are bonded together. The fixture and mirrors are left overnight to allow the epoxy to cure. The overnight cure is necessary so that the epoxy has the right amount of plasticity while still providing enough strength to hold the mirror to the adjustable fixture. If too rigid of an epoxy is used for this step, internal stresses may build in the epoxy used to bond the mirrors together 
(along the bonding seam), while using an epoxy that isn't rigid enough may cause the mirror alignment to change more when bonding. Afterwards, the shims are taken out and the mirrors are aligned once again. The mirrors are now ready to be bonded as described in the Section 3.

\subsection{Master Retroreflector}

The other fixture used to bond the hollow retroreflectors consisted of a solid corner cube as a mandrel. Glass panels are placed on top of the mandrel and then bonded together (Fig. 2). The master cube has its corners beveled so that any bonding material that wicks through the bonding seam will not get in contact with the master cube and cause the glass panels to bond to it.

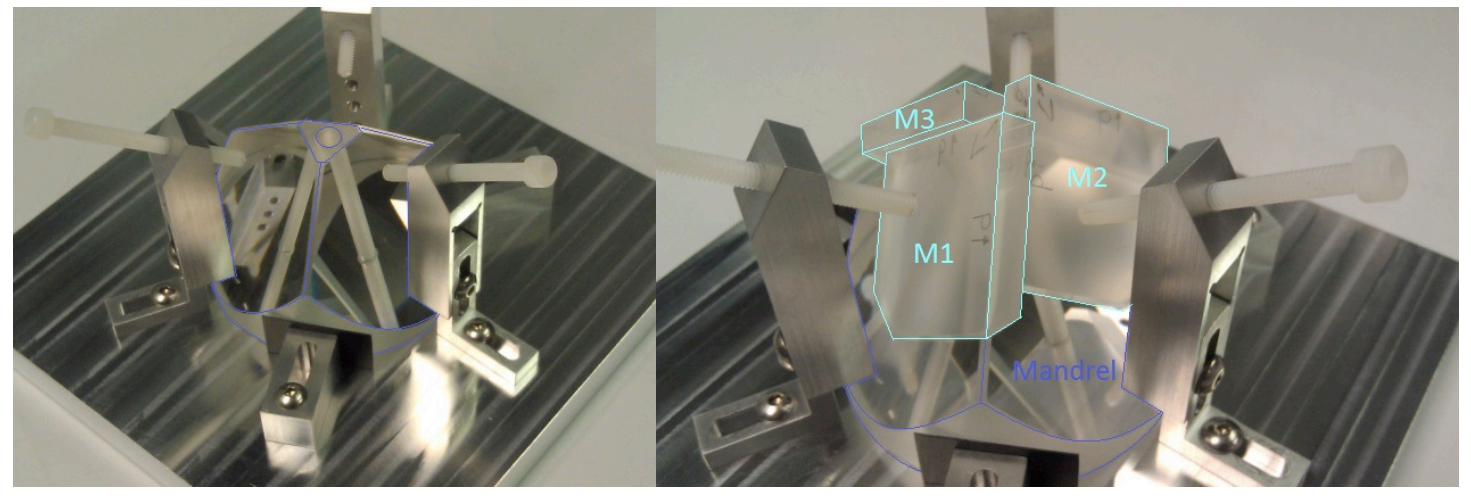

(a)

(b)

Fig. 2 (a) Mandrel situated before the glass panels are placed, and (b) after the glass panels are secured. In this case, the entire thickness of the bonding surface is placed on the adjacent glass panel.

The glass panels and master cube are both cleaned with vinegar and then cleaned again with acetone to remove any residue. Although vinegar was used, any mild acid should suffice. The glass panels are placed on the master cube and securely held with screws that have rounded tips. Extreme cleanliness and care is needed when initially placing the panels or scratches on either surface may occur. Alignment is done by pressing the glass panels both against the master cube 
and against each other. The accuracy to which the panels are aligned is judged by monitoring the resulting interference fringes and are adjusted until the fringes are minimized. The screws are then tightened to securely hold the glass panels in place. The interference fringes should be monitored overnight to check that the glass panels are not slipping or changing position. Afterwards, either the epoxy or hydroxide solution can be applied. After the mirrors have been bonded, the mirror coating will need to be applied to the corner cube surface.

\section{Bonding Procedure}

The bonding methods described in this section can be used for either bonding fixture. Some adjustments to the procedure can be done, but using a brush to paint either the epoxy or hydroxide solution proved to be an extremely successful technique.

\subsection{Epoxy Bonding}

In general, only a thin bonding seam is used when bonding a hollow retroreflector using an epoxy. A mirror overlap of approximately $1.3 \mathrm{~mm}$ can be used to bond hollow retroreflectors with sub-arcsecond dihedral angle errors and still provide sufficient strength for most uses (see Fig. 1b). To do this, a rough bonding surface sits on top of a mirrored surface and the epoxy is applied to the back of the bonding seam with a brush. The epoxy will wick into the bonding seam to fill the gap. Applying the epoxy at the bottom of the mirrors will cause the retroreflectors to "flower open" as the epoxy cures. The amount to which this happens varies depending on what epoxy is used and how much is applied. The rough surface is needed for the epoxy to retain its strength and the thin bonding seam provides enough rigidity without significant internal stresses building up within the bond itself ${ }^{13}$. If significant internal stresses 
are trapped within the bond, they will slowly be worked out through the movement of voids and dislocations over time which may cause the shape of the retroreflector to change as well ${ }^{14}$.

When using the master retroreflector fixture, the epoxy can be easily applied using a brush and a minimum amount of epoxy should be applied in an even manner. This can often be done by slowly dragging the brush over the bonding seam a few times. Using a significant amount of epoxy will cause the corner cube to flower open more than when less epoxy is used. Once the epoxy is applied, the glass panels should stay on the fixture until the epoxy has cured.

If epoxy bonding is used with the adjustable fixture, the epoxy should be applied minimally and in an even manner as well. As the epoxy begins to cure, the corner cube will flower open as can be seen with the interferometer. While the epoxy is in its curing phase the dihedral angles can be adjusted. The amount of time the mirrors can be adjusted depends on the epoxy, but care should be taken not to adjust for too long or stresses may build up within the bond. For the corner cubes presented in Table 1, an Epon/Versimid epoxy in a 50/50 mixture was used. It typically took 2-3 hours before the mirrors would begin to change shape, and then adjustments were made for an additional 3-5 hours. The epoxy then cured overnight and a razor was used to remove the Urethane that bonded the mirrors to the fixture. Once this was done, the corner cube could be removed from the fixture.

By situating the mirrors in the adjustable fixture with an approximate $1.3 \mathrm{~mm}$ bond thickness and using Epon/Versamid epoxy, the authors were able to routinely fabricate hollow retroreflectors with dihedral angle errors typically less than a few arcseconds, and in some instances, sub-arcsecond errors (see Table 1). 


\subsection{Hydroxide Bonding}

Although using an epoxy has been successful in bonding hollow corner cubes, the high CTE of most epoxies makes it difficult for the corner cube to retain its shape when subjected to significantly different temperatures ${ }^{3}$. Although this distortion can be lessoned through the use of a tongue-and-groove design ${ }^{2,4}$, another potential method may be to use HCB. The HCB process works by putting a small amount of hydroxide solution between two glass surfaces. The hydroxide solution etches the surfaces that then form glass chains within the solution. As the liquid evaporates, the chains form a rigid structure that bonds the surfaces together which form a quasi-monolithic structure. For a more complete description of the HCB process, see Ref. 15 and Ref. 16. The resulting bond is one that is strong, thin, and essentially glass. Because of this, the bond will be one that produces less distortion in the corner cube when compared to epoxy as the corner cube is thermally cycled ${ }^{3}$. For this reason, HCB was chosen to determine the feasibility of being used for bonding hollow corner cubes.

Using the epoxy bonding technique described in Sec. 3.1 with HCB proved to be ineffective. When the retroreflectors were bonded using a $1.3 \mathrm{~mm}$ bonding seam, the corner cubes would either flower open until they could not be measured by the interferometer, or they fell apart after a few days. Leaving the retroreflector to cure for one or two weeks reduced the amount to which the retroreflectors would flower out after being removed from the fixture, but the retroreflectors that bonded without large dihedral angle errors typically lacked any significant strength and could be pulled apart with little effort.

Increasing the bond thickness such that the entire width of the mirror was on the mirrored surface drastically improved the results, although the dihedral angle errors were typically an order of magnitude worse than when an epoxy was used. Using the same procedure from one 
retroreflector to the next would often produce drastically different results and the authors attribute this to the rough bonding surface that was used. Although it has been shown that using $\mathrm{HCB}$ on rough surfaces can produce similar strengths to those when polished surfaces are used ${ }^{10}$, the thickness of the bond is less than the root-mean-square (rms) roughness of the bonding surface which will cause bonds to form randomly on the bonding surface which, in turn, cause the mirrors to shift their alignment by "pivoting" on the peaks of the ground surface finish.

To remedy these problems, the bonding surface of the mirrors was changed from a ground finish to a flat and smooth surface. These mirrors were made by taking a solid retroreflector (Edmund Optics, part number 49-011) and cutting a $6.25 \mathrm{~mm}$ slice from the corner cube and then coated with a protective aluminum layer. Significantly better results were achieved when the entire $6.25 \mathrm{~mm}$ bonding width of the flat and smooth surface was used (see Table 1).

To bond the mirrors, a brush was dipped into a sodium silicate solution that had been volumetrically diluted by a factor of 1:4 with a stock solution (Sigma Aldrich, part number 338443) and applied to the back of the bonding seam. The solution would then wick in to fill the bonding area. The retroreflector was left alone for twenty one days and then removed from the fixture. When taken out of the fixture, there is often a small change in the dihedral angle errors of the retroreflector, and there may be some drift over the course of a few days.

Although using a brush to administer the hydroxide solution showed promising results, it was difficult to apply the same amount of solution each time. Applying the same amount to each bonding surface is critical so that the mirrors will change in a uniform manner when the bond is curing. When using larger bonding surfaces, it may be useful to apply the solution using a micropipette. In this manner, the amount of solution that is applied can be controlled in a more precise manner. 


\section{Results}

A total of ten hollow retroreflectors were made using HCB and epoxy bonding methods. Seven retroreflectors were made using the adjustable fixture, and three were made using the mandrel. In all cases where HCB was used, the bonding area was a flat and smooth surface as described above. Although a bonding surface with a ground finish can be used (as was done with the epoxy bonded corner cubes), the resultant dihedral angle errors are significantly larger than when a flat and polished surface is used ${ }^{3}$.

\begin{tabular}{|c|c|c|c|c|c|c|}
\hline Retroreflector & $\begin{array}{l}\text { Fixture } \\
\text { Method }\end{array}$ & $\begin{array}{l}\text { Bonding } \\
\text { Method }\end{array}$ & $\begin{array}{l}\text { Aperture } \\
(\mathbf{m m})\end{array}$ & $\begin{array}{l}\text { Dihedral } \\
\text { Angle Error } \\
\text { (arcsec) }\end{array}$ & $\begin{array}{l}\text { Wavefront } \\
\text { (PV@633 } \\
\text { nm) }\end{array}$ & $\begin{array}{l}\text { Max. Beam } \\
\text { Deviation } \\
\text { (arcsec) }\end{array}$ \\
\hline 1 & Adjustable & $\mathrm{HCB}$ & 40 & $\begin{array}{l}-1.9,0.3,- \\
8.4\end{array}$ & 2.92 & 19.5 \\
\hline 2 & Adjustable & $\mathrm{HCB}$ & 40 & $\begin{array}{c}-2.9,-10.4,- \\
13.4\end{array}$ & 8.28 & 35.2 \\
\hline 3 & Adjustable & Epoxy & 40 & $\begin{array}{c}-0.1,0.0,- \\
1.4\end{array}$ & 1.28 & 5.8 \\
\hline 4 & Adjustable & Epoxy & 40 & $\begin{array}{c}-0.9,0.0,- \\
1.8\end{array}$ & 1.49 & 6.6 \\
\hline 5 & Adjustable & Epoxy & 40 & $\begin{array}{c}0.4,3.7 \\
2.8\end{array}$ & 2.97 & 12.9 \\
\hline 6 & Adjustable & Epoxy & 40 & $\begin{array}{c}3.6,0.3 \\
3.7\end{array}$ & 2.60 & 13.0 \\
\hline 7 & Adjustable & $\begin{array}{l}\text { Epoxy }+ \\
\text { tongue and } \\
\text { groove }\end{array}$ & 115 & $\begin{array}{c}1.6,-1.7,- \\
0.9\end{array}$ & 2.55 & 5.4 \\
\hline 8 & Mandrel & $\mathrm{HCB}$ & 40 & $\begin{array}{c}1.9,1.7 \\
3.1\end{array}$ & 1.46 & 10.5 \\
\hline 9 & Mandrel & $\mathrm{HCB}$ & 40 & $\begin{array}{c}1.98,2.32 \\
-0.50\end{array}$ & 0.91 & 8.61 \\
\hline 10 & Mandrel & $\mathrm{HCB}$ & 40 & $\begin{array}{c}0.93,0.52 \\
1.58\end{array}$ & 0.79 & 6.10 \\
\hline
\end{tabular}

From Table 1, it can be seen that there is a noticeable difference in the physical properties of Retroreflectors 1 and 2. This is most likely because a slightly different method was used that incorporated the reversible nature of $\mathrm{HCB}^{15,16}$. By applying a small amount of solution one day, letting it cure overnight, and then adding more solution the next day, stresses and misalignments 
from the bonds can be alleviated while forming additional bonding area at the same time since there are more hydroxide ions now available. In this manner, misalignments of the mirrors from the bonding process can be taken out while adding additional bonding area. Unfortunately, when this is done, the change in room temperature from day to day may have caused the fixture to be in a different orientation the next time the solution was added. This, in turn, may have caused the mirrors to cure in a different position from the previous day. It is believed that this effect is what led to the larger values of the second retroreflector, although more statistics are needed before a definitive conclusion can be made.

As can be seen from Retroreflectors 3-6, using epoxy to bond the corner cubes is a very effective method. In all cases the dihedral angle errors are less than a few arcseconds and in several cases, one or two dihedral angle errors were less than 1.0 arcsecond.

\subsection{Scaling-up the Retroreflector Size}

A benefit of using the adjustable fixture is that it allows for scaling-up of the retroreflector size in an easy manner while still obtaining similar results to the smaller retroreflectors. To demonstrate this, a hollow retroreflector with an approximate $115 \mathrm{~mm}$ aperture was made from single crystal silicon (SCSi) mirrors using a Stycast epoxy. The mirrors used a tongue-andgroove design such that a $2.54 \mathrm{~mm}$ by $2.54 \mathrm{~mm}$ groove was machined into the SCSi mirrors (see Fig. 3). The tongue-and-groove design was chosen for this particular corner cube because this retroreflector will potentially be used as a prototype for the CIRS-lite instrument and the corner cube will operate at lower temperatures ${ }^{17}$. The tongue-and-groove design has demonstrated fewer distortions when used at low temperatures when compared to only a $1.3 \mathrm{~mm}$ overlap $^{2}$. Although this design was chosen, the same method used for Retroreflectors 3-6 could also be used. 
Stycast epoxy was applied with a brush such that it filled the groove and then the mirrors were assembled and aligned in the fixture. Urethane was applied in small amounts to bond the edges of the SCSi mirrors to the fixture. The mirrors were realigned every 20-30 minutes for approximately five hours and then left to cure overnight. The retroreflector was then taken out of the fixture the next day and the resulting physical properties are shown in Table 1.

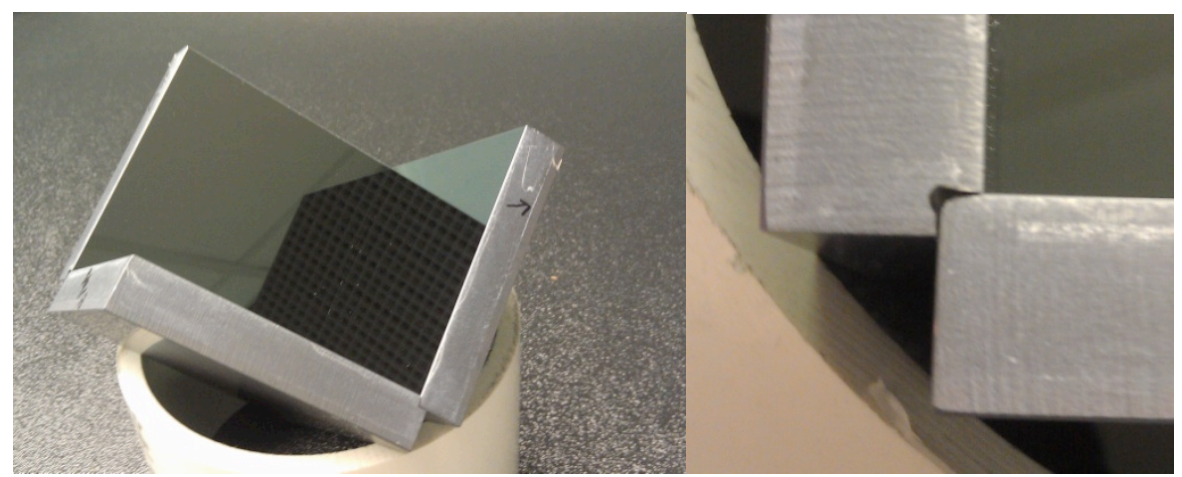

(a)

(b)

Fig. 3 (a) A $115 \mathrm{~mm}$ aperture SCSi hollow retroreflector made using the adjustable fixture, and (b) a close-up of the groove used to bond the retroreflector.

\subsection{Master Retroreflector Method}

In addition to making hollow retroreflectors using an adjustable fixture, three retroreflectors were made using a solid master as a mandrel. The mandrel was made of BK7 glass, has a 76.2 $\mathrm{mm}$ aperture, and dihedral angle errors less than 1.0 arcsecond. Glass panels cut from solid corner cubes were placed around the mandrel and held in place through the use of screws (see Fig. 2). A significant amount of pressure is usually required to adjust the panels such that the interference fringes are minimized and the lack of mirrored coating allows this to happen without scratching the surfaces. Moreover, a mirrored surface all too often will wash out the interference fringes, making them difficult or impossible to see. 
A small amount of hydroxide solution was then applied with a brush along the outside bonding seams of the retroreflector. If too much solution is used, the excess may leak into the inside of the retroreflector which could form a fillet and either distort the far field diffraction pattern, or cause the aluminum coating to be uneven or not adhere properly. Additional solution can be applied later on if too little solution is initially applied. It should be noted that it may not always be possible to add additional solution since a fillet tends to form along the bonding seam which can inhibit the amount of solution that wicks into the bonding area.

After the solution was added, the retroreflectors were left to cure for twenty days. During this time, the interference fringes changed in response to the daily change in room temperature, but no additional change could be inferred from the bonding process, even when additional solution was intermittently added over two days. The changing fringes are most likely due to the difference in CTE of the BK7 mandrel and the fused silica panels. Once the curing period was over, the corner cube was taken off and the aluminum coating was then applied. The resulting retroreflectors have physical properties shown in the last three rows of Table 1.

It can be seen from Table 1 that using $\mathrm{HCB}$ with the mandrel produced more precisely aligned corner cubes than when the adjustable fixture was used. This is most likely due to the fact that the adjustable fixture breathes significantly while the bonds are curing, but more statistics are needed before a definitive conclusion can be made.

\section{Discussion}

It can be seen from Table 1 that both the adjustable fixture and the mandrel are capable of producing well-aligned corner cubes. In this section, a discussion of each fixture is presented along with potential improvements. 


\subsection{Adjustable Fixture}

The nature of the adjustable fixture suits itself well for being used in a mass-production setting. The repeatable nature to which corner cubes can be bonded, the ease to which the bonding takes place, the ability to be used for a range of corner cube sizes, and the ease to which the fixture can be modified make it ideal for repeated use. The biggest advantage that the adjustable fixture has over the mandrel is that the adjustable fixture allows for the dihedral angle errors to be easily adjusted. In some applications, such as next-generation retroreflectors for lunar laser ranging, an offset in one or more of the dihedral angle errors will need to be present ${ }^{18}$. Even though this could be done with the mandrel, how well the dihedral angles are set will be determined by how well the offset is polished into the mandrel.

Although the hollow retroreflectors made using HCB were not as good as those when epoxy

was used, the authors believe that additional improvements to the design of the fixture and bonding surface may give improved results. The temperature stability of the room when bonding the retroreflectors was typically $\pm 2 \mathrm{C}$, but could be as high as $\pm 3.5 \mathrm{C}$. This would cause the aluminum fixture to "breath" while the temperature in the room oscillated. By using mirrors that had been epoxied to the fixture, but not yet bonded together, it was determined that a $\pm 2 \mathrm{C}$ change in the air temperature of the room could produce a change in dihedral angle errors as much as \pm 5 arcseconds, and changes in the wavefront by as much as 2 waves (at $633 \mathrm{~nm}$ ). Although a more stable air temperature would be beneficial, a more suitable remedy would be to make the fixture out of an ultra-low expansion material such as ULE or Zerodur. This would greatly reduce the amount to which the fixture changed when bonding with HCB.

While the mirrors that were cut from a solid corner cube produced promising results, the exact perpendicularity between the mirrored surface and the bonding surface was not precisely 
known to be better than 3 arcseconds. Having mirrors with a known perpendicularity, and less than 1 arcsecond should help to improve the accuracy to which the hollow retroreflectors are bonded when using $\mathrm{HCB}^{19}$.

Additionally, changes to the mirror design may also improve the bonding technique. In one case, when the rough bonding surfaces were initially being tested for use with $\mathrm{HCB}$, a groove 1.3 $\mathrm{mm} \times 1.3 \mathrm{~mm}$ was machined down the center of each bonding surface and $0.15 \mu \mathrm{L}$ of sodium silicate solution was applied with a micropipette to the top of the groove which caused the solution to spread from the center of the bonding surface to the edges. In this case, the dihedral angles changed significantly less than without the groove. The authors believe that this may have to do with how the solution is applied. When the solution is applied to the back of the bonding seam, a fillet forms where the two mirrors are in contact. It is believed that this fillet can cause the dihedral angles to become skewed. When a groove is machined down the middle of the bonding surface, two fillets will form, but the effects from each one should cancel the other out. By using a smaller groove down the center of a polished bonding surface, the authors believe that greater precision could be achieved when bonding.

\subsection{Solid Mandrel}

The mandrel fixture is well-suited when only a few corner cubes need to be made in an inexpensive manner. The total cost of the mandrel fixture was approximately a factor of five less while still being able to produce precision-bonded corner cubes. This was due to the fact that many of the components needed are easily machined or "off-the-shelf."

The primary disadvantage of using the mandrel is that the alignment of the retroreflector will be limited by the mandrel itself. If an offset in only one of the dihedral angles is needed, then a completely new mandrel will need to be polished. Anecdotal evidence also seems to suggest that 
using the same mandrel repeatedly may produce corner cubes whose dihedral angles errors are bonded with less precision.

Although promising results were achieved using the mandrel, further improvements may produce better outcomes. Using a mandrel made of the same material or CTE of the panels may help to improve the final alignment since daily changes in the room temperature will cause the mandrel and panels to change by differing amounts. When using HCB, panels with a perpendicularity better than one arcsecond should also help to improve results since the exact perpendicularity of the mirrors used was only known to be better than 3 arcseconds.

\section{Conclusion}

A total of ten hollow retroreflectors were made using two different bonding fixtures. One bonding fixture consisted of adjustable panels that allowed for real-time alignment of the corner cube mirrors while the other used a solid corner cube as a mandrel that glass panels can be placed on top of and held in place through screws. Although both methods were able to produce hollow corner cubes with dihedral angle errors of less than a few arcseconds in a repeatable manner, each method presents itself for different uses. The adjustable fixture lends itself to being used for mass production of corner cubes since the wear and tear of the fixture is minimal, while the mandrel fixture presents itself for being used when only a few corner cubes are needed. Modifications to each fixture were also suggested to be able to produce hollow corner cubes with more precisely bonded mirrors.

In addition to using two bonding fixtures, two bonding methods were used to construct the hollow retroreflectors. Although epoxy bonding was not used with the mandrel fixture, the use of epoxy bonding with the adjustable fixture proved to be very effective when constructing 
hollow corner cubes and routinely produced corner cubes with sub-arcsecond dihedral angle errors.

Although the HCB method did not produce corner cubes as precisely bonded as when epoxy was used, the larger dihedral angle errors are attributed to both using fixtures that changed considerably while the curing process was taking place as well as have mirror panels that were not polished to the necessary perpendicularity required to achieve sub-arcsecond dihedral angle errors. Corner cubes made using the mandrel method were bonded with dihedral angle errors that had the same 3 arcsecond precision of the mirror panels, thus implying a limitation of the mirror panels and not the bonding fixture. Using a fixture made out of a low CTE material along with mirrors that have a bonding surface and mirror surface perpendicular to better than 1 arcsecond, one should be able to produce hollow corner cubes as good or better than when epoxy bonding was used.

\section{Acknowledgments}

The authors would like to thank Peter Hill and Felix Threat, both of the optics branch at Goddard Space Flight Center. Both were instrumental in fabrication methods and coating techniques for the mirror panels used to construct the corner cubes. The authors would also like to thank Jeffrey Livas of Goddard Space Flight Center whose insight and comments were greatly appreciated while formulating this paper.

\section{References}

1. R. J. Hill, T. L. Courtney, S. D. Park, and D. M. Jonas, “Lightweight hollow rooftop mirrors for stabilized interferometery," SPIE Opt. Eng. 50 (10), 105103 (2013). 
2. J. Lyons, and P. Hayes, "High-optical-quality cryogenic hollow retroreflectors," Proc. Of SPIE, 2540, 94-100 (1995).

3. A. Preston, and S. Merkowitz, "Next generation hollow retroreflectors for lunar laser ranging," Appl. Opt., 52, 36, 8676-8684 (2013).

4. P. R. Yoder Jr., Mounting Optics in Optical Instruments $2^{\text {nd }}$ ed., 353-397, SPIE Press Monograph, Vol. PM181, Bellingham, Washington, 2008.

5. D. Gwo, S. Wang, K. Bower, D. Davidson, P. Ehrersberger, L. Huff, E. Romero, M. Sullivan, K. Triebes, and J. Lipa, “The Gravity Probe-B Star-Tracking Telescope,” Adv. In Space Research, 32, 1401-1405 (2003).

6. P. Mammini, “A bonded precision optical assembly using potassium hydroxide.” Optical Materials and Structures Technologies IV, 7425, pg. 742500 (Proc. of SPIE).

7. A. Preston, and G. Mueller, "Bonding SiC to SiC Using a Sodium Silicate Solution," Int. J. Appl. Ceram. Tech., 9, 764-771 (2012).

8. E. Elliffe, J. Bogenstahl, A. Deshpande, J. Hough, C. Killow, S. Reid, D. Robertson, S. Rowan, and G. Cagnoli, "Hydroxide-catalysis bonding for stable optical systems for space," Class. Quant. Grav., 22, S257-S267 (2005).

9. J. SanJuan, R. Spannagel, D. Korytov, A. Preston, J. Livas, C. Braxmaier, and G. Mueller, "Silicon carbide telescope dimensional stability for space-based gravitational wave detectors," Rev. Sci. Instr., (2012).

10. A. Preston, B. Balaban, and G. Mueller, "Hydroxide-Bonding Strength Measurements for SpaceBased Optical Missions," Int. J. Appl. Ceram. Tech., 5, 365-372 (2008).

11. N. Beverage, A. van Veggel, M. Hendry, P. Murray, R. Montgomery, E. Jesse, J. Scott, R. Bezensek, L. Cunningham, J. Hough, R. Nawrodt, S. Reid, and S. Rowan, "Low-temperature strength tests and SEM imaging of hydroxide catalysis bonds in silicon," Class. Quant. Grav., 28, 085014 (2011).

12. Zygo Corp., "Corner Cube MetroPro Application," can be found at http://www.zygo.com/?sup=resource/manuals.cgi?overview=GPI. 
13. A. F. Abdelkader, and J. R. White, "Curing characteristics and internal stresses in epoxy coatings: Effect of crosslinking agent," J. Material Sci., 40, 8, 1843-1854 (2005).

14. T. S. Lundstrom, and B. R. Gebart, "Influence from process parameters on void formations in resin transfer molding," Polymer Composites, 15, 1, 25-33 (1994).

15. D. Gwo, "Ultra-precision bonding for cryogenic fused-silica optics," SPIE Conference on Cryogenic Optical Systems and Instruments VIII," 3435, 136-142 (1998).

16. United State Patent, "Hydroxide-Catalyzed Bonding.” (2003), patent number US 6548176 B1.

17. J. Brasunas, M. Abbas, V. Bly, M. Edgerton, J. Hagopian, W. Mamakos, A. Morell, B. Pasquale, and W. Smith, "CIRS and CIRS-lite designed for the outer planets: TSSM, EJSM, JUICE,” International Workshop on Instrumentation for Planetary Missions, (2012).

18. T. Otsubo, H. Kunimori, H. Noda, H. Hanada, H. Araki, and M. Katayama, “Asymmetric dihedral angle offsets for large-size lunar laser ranging retroreflectors," Earth Planets Space, 63, e13-e16 (2011).

19. C. Killow, E. Fitzsimons, J. Hough, M. Perreur-Lloyd, D. Robertson, S. Rowan, and H. Ward, "Construction of rugged, ultrastable optical assemblies with optical component alignment at the few microradian level," Appl. Opt., 52, 2, 177-181 (2013).

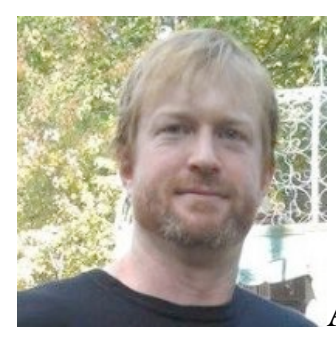

Alix Preston performed this work while a NASA Postdoctoral Fellow at Goddard Space Flight Center. He received his BS degree in engineering physics from the Colorado School of Mines in 2004, and his Ph.D degree in physics from the University of Florida in 2010. Much of his work focuses on using hydroxide catalysis bonding for precisionaligned optics for space-based missions. 
Stephen Merkowitz is a physicist and project manager at NASA Goddard Space Flight Center. $\mathrm{He}$ received a $\mathrm{BA}$ in physics from the University of Colorado and a $\mathrm{PhD}$ in physics from Louisiana State University. He currently manages NASA's Space Geodesy Project and is the Principle Investigator for the Global Positioning System (GPS) Laser Retroreflector Array instrument.

\section{Caption List}

Fig. 1 (a) Concept of the adjustable fixture, and (b) an illustration of the bonding seam when epoxy was used as a bonding agent.

Fig. 2 (a) Mandrel situated before the glass panels are placed, and (b) after the glass panels are secured. In this case, the entire thickness of the bonding surface is placed on the adjacent glass panel.

Fig. 3 (a) A $115 \mathrm{~mm}$ aperture SCSi hollow retroreflector made using the adjustable fixture, and (b) a close-up of the groove used to bond the retroreflector.

Table 1 Physical properties of the retroreflectors made using the adjustable fixture and the solid mandrel. 